\title{
Reformist Framework for Improving Human Security for Mobile Robots in Industry 4.0
}

\author{
Anand Singh Rajawat $\left(\mathbb{D},{ }^{1}\right.$ Pradeep Bedi $\left(\mathbb{D},{ }^{2}\right.$ S. B. Goyal $\left(\mathbb{D},{ }^{3}\right.$ Piyush Kumar Shukla $\left(\mathbb{D},{ }^{4}\right.$ \\ Atef Zaguia $\mathbb{D}^{5},{ }^{5}$ Aakriti Jain $\mathbb{D}^{6},{ }^{6}$ and Mohammad Monirujjaman Khan $\mathbb{D}^{7}$ \\ ${ }^{1}$ Department of Computer Science Engineering, Shri Vaishnav Vidyapeeth Vishwavidyalaya, Indore, India \\ ${ }^{2}$ Lingaya's Vidyapeeth, Faridabad, India \\ ${ }^{3}$ City University, Petaling Jaya, Malaysia \\ ${ }^{4}$ Computer Science \& Engineering Department, University Institute of Technology, Rajiv Gandhi Proudyogiki Vishwavidyalaya, \\ (Technological University of Madhya Pradesh), Bhopal 462033, India \\ ${ }^{5}$ Department of Computer Science, College of Computers and Information Technology, Taif University, Taif 21944, Saudi Arabia \\ ${ }^{6}$ Department of Computer Science and Engineering, Sagar Institute of Research and Technology Excellence, Bhopal, \\ Madhya Pradesh, India \\ ${ }^{7}$ Department of Electrical and Computer Engineering, North South University, Bashundhara, Dhaka 1229, Bangladesh
}

Correspondence should be addressed to Mohammad Monirujjaman Khan; monirujjaman.khan@northsouth.edu

Received 17 July 2021; Revised 11 September 2021; Accepted 17 September 2021; Published 1 October 2021

Academic Editor: Sikandar Ali

Copyright (C) 2021 Anand Singh Rajawat et al. This is an open access article distributed under the Creative Commons Attribution License, which permits unrestricted use, distribution, and reproduction in any medium, provided the original work is properly cited.

\begin{abstract}
In this paper, the cooperation between human and robot companies plays a significant role in factories, contributing to greater productivity and efficiency. However, this development breaches established safety procedures when the workspaces are separated from the robot and the human being. These changes have been reflected in industrial robotic safety standards for the last 20 years. We have directed the expansion of a broad field of examination, which focuses on avoiding robotic humans' effects and minimizing associated risks and consequences. The paper depicts an analysis of prominent safety systems projected and implemented in engineering robotic surroundings that contribute to safe, collective work between humans and robots. Besides, the current regulation has introduced a review and new concepts. The discussion includes multidisciplinary approaches such as estimating and evaluating human-robot collision injuries, mechanical equipment and software to minimize human-robot impacts, impact detection systems, and collision prevention strategies and minimizing their impact to proposed approach for Human Security with Mobile Robots in Industry 4.0 using SDN and CPS with GMM-GM machine learning model.
\end{abstract}

\section{Introduction}

The rapidly increasing new challenges have led to the new industrial revolution to make manufacturing processes more productive, independent, and user-friendly. An original industry concept, Industry 4.0 [1], has emerged that today refers to current manufacturing technology automation and data exchange trends through creating a "smart factory." The continuous growth in cyber-physical systems IoT and SDN increased computing ability is the foundation of Industry 4.0. In Figure 1, we represent the nine impotent pillars of industry 4.0 [2]. The robotic processes are remotely associated with the computer system, which meets the hardware and software requirements. Our job is to provide an intelligence service to the Mobile Robot to establish the connection between the machines. Figure 1 represents Current Application Industry 4.0 [3]. In this regard, we offer a direction-finding design based on integrated control, which monitors robotic navigation and establishes the connection between the machines [4]. Four phases include autonomous Mobile Robot navigation: mapping, location, design, and implementation. 


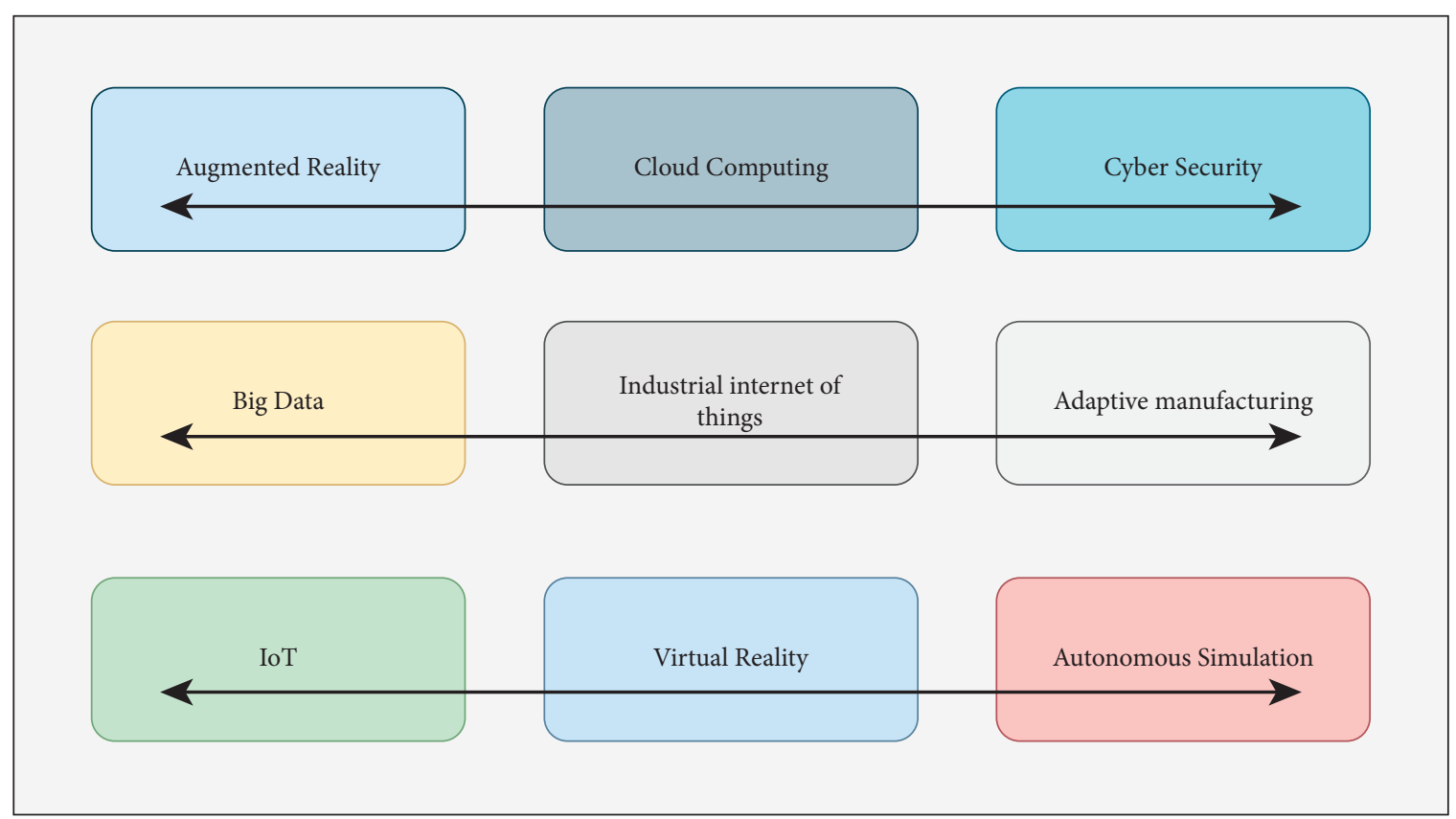

FIgURE 1: The nine components of Industry 4.0.

Therefore, it is sensible to know the environment map before starting the designing phase [5]. As there are numerous methods available to work with robots, we are not going to deal with this problem in the planning and executing of robots. Finally, concerning the classification [6] of the navigation architecture commonly used in the literature, the research paper's architecture can be seen as a mixture of many existing systems: it is primarily planned with subtle fundamentals for tackling difficulties and sudden changes in the environment. The several issues tackled in the designing framework of Industry 4.0 are the nonexistence of concurrent encryption acquiescent to fissures and security breaches and the lack of developed infrastructure that meets Industry 4.0. To fill this security-related gap, upgradation of infrastructure is needed within few years.

\section{Related Work}

Chen et al.'s [7] discussion includes multidisciplinary approaches such as estimating and evaluating human-robot collision injuries, mechanical equipment and software to minimize human-robot impacts, impact detection systems, and collision prevention strategies as well as reducing their impact. Wan et al. [8] represent the outline of IoT and Industry 4.0 because the industry is changing rapidly, and the main objective is to enhance the quality of industry infrastructure and resources. Åblad et al.'s [9] studies have shown that both practices recover industrial wireless sensor networks' dependability when interference occurs. Agarwal et al.'s [10] paper can help you deeply understand the current application of data management in related industries and identify interesting open research opportunities. In industrial environments, Chiang et al. [11] presented an overall Mobile Robot navigation methodology that uses automation to support open-loop robotic behaviour and specifications.
Oztemel and Gursev's [12] paper describes a recently designed architecture for IoT-based networks used for the prominent use of physical layer, network layer, and application layer. In Rajawat et al. [13], simulations show the advantage of reasoning developed IoT and show that CACR can suggestively increase energy efficacy and cost optimization for handling materials. Evjemo et al. [14] presented a program in which robots never intersect and accomplish maximum intervals equivalent to those produced that allow harmonization to be generated. Table 1 shows the comparison of studies for different frameworks and Table 2 shows the comparison of applications of Industry 4.0 for various domains.

\section{Reformist Framework}

The controller can be reconfigured and the production functions can be extended. Modular production units proposed a modular-adaptive autonomous robotic $[15,16]$ island to improve the workshop's assembly capacity from the point of view of modular production units. Besides, an integrated management framework has enhanced flexible manufacturing skills, which controls and organizes a modular manufacturing unit-which proposed that cognitive robots be vertically integrated into production physically by the cyber-the vertical integration of the cognitive robots in the manufacturing industry and the coordination with the manufacturing system. Cognitive robots can perceive information uncertainty in the context of smart production, change schedules, and adjust production behavior to face a complex production issue independently. Therefore, the intelligence of robot units is essential. The following are several suggestions for building modular production units. Furthermore, the interaction heterogeneity should be considered. The functions of various 
TABLE 1: Comparison of studies for different frameworks.

\begin{tabular}{lccc}
\hline S. no. & Study & Framework name & Highlights \\
\hline 1 & Sanz et al. 2021 & BiDrac Industry 4.0 framework & $\begin{array}{c}\text { Designed to integrate various activities of CASE technologies } \\
\text { Designed to operate in harsh and hazardous environments } \\
\text { Designed to locate numerous warehouses, data centres, and } \\
\text { supply chain operations }\end{array}$ \\
3 & Di Castro et al. 2018 & RoboPlanner & $\begin{array}{c}\text { Designed as software stack which allows customers to request } \\
\text { deliveries }\end{array}$ \\
\hline
\end{tabular}

TABle 2: Comparison of applications of Industry 4.0 for various domains.

\begin{tabular}{lccc}
\hline S. no. & Study & Domain & Highlights \\
\hline 1 & Chen et al. 2021 & Reinforcement learning & Application of reinforcement learning used in classification problems \\
2 & Fatorachian et al. 2021 & Supply chain management & $\begin{array}{c}\text { To optimize the prominent factors used in supply chain management } \\
\text { The improvements implemented in manufacturing industry with help } \\
3\end{array}$ \\
Ammar et al. 2021 & Manufacturing industry & of Industry 4.0 frameworks \\
4 & Ralph et al. 2021 & Manufacturing industry & Major advantages of principles of Industry 4.0 in metal forming \\
\hline
\end{tabular}

modular production units for a particular product may be redundant, and therefore an optimal or inappropriate combinatorial system is crucial. Each production plant can comply with product manufacturing requirements and selforganize to increase intelligent plant efficiency. Small quantities of various products are disorderly brought into the fabrication system during intelligent manufacturing, leading to an impasse. The approach to preventing impasse in flexible production systems with human security with Mobile Robots in Industry 4.0 [17] is currently a hot research topic. This would make it possible for the production unit to adapt quickly to changes in operating conditions. Use the available software components to adjust the robot to changing operating conditions and upgrade the robot function. Besides, an advanced robot controller design has been introduced that can be used in the new generation of robots. Models of control unit components have been developed for component model since standardization and universality can improve reconfiguration processes. This would make it possible for the production unit to adapt quickly to changes in operating conditions. Use the available software components to adjust the robot [18] to changing operating conditions and upgrade the robot function [19]. Besides, an advanced robot controller design has been introduced that can be used in the new generation of robots for Reformist Framework for improving human security with Mobile Robots in Industry 4.0-developed Reformist Framework for improving human security with Mobile Robots, a new distributed multiagent controller system that is compatible with an intelligent and reconfigurable number control computer (CNC).

The specific implementation steps are as follows:

Step-1: Initialize the starting point [Open list].

Step-2: If Open list: Null Search Failure

Step-3: If node-value: least-value Open List=current node

Step-4: If node: target-point: Search ends.
Step-5: Expand the node Move in a circular and straight path.

Step-6: If obstacle $=$ present, go to step 5

Step-7: Change motion if exact type of, motion not found.

Step-8: Jump to next open list node.

3.1. Mobile Robots (MR) in Smart Factories. Mobile Robot (MR) systems are embedded in intelligent factories with sensors in safe working areas to collaborate with human robots. Compared to traditional industrial robots [20], the Mobile Robot (MR) has several advantages. There are many advantages of Mobile Robot (MR). These robots provide people with safe room and fence protection, and they can provide room for traditional robots. Proximity sensors are used to decrease the speed of robots when people approach it, the limitation of forces to minimize human and environmental risks, and human intent and management accordingly. The prominent ideology is that Mobile Robots (MR) do not harm a person and that controlled force and speed are the means to protect someone. Separation monitoring, hand guidance, and monitored stop-office at safety rates. Planning and control of vision and CAD robots help to avoid long-term manual robot programming. Dynamic motion primitives to parameterize Mobile Robot (MR) motion can also relieve learning by demonstrating the need for manual programming. This will also provide greater flexibility where, for example, changes in production require the plant's interior.

3.2. Cyber-Physical Systems (CPS). Cyber-physical systems (CPS) is one of the significant contributing factors involved in Industry 4.0. Cyber-physical systems is connected to physical devices with the help of the internet (internet of things), which depicts that the entire physical and virtual worlds are connected due to CPS. The physical world 
contains computer and machinery, cyber-spaces contain applications, software, routines, and analytics activities, and communication network is the interconnection between the physical world and software. The CPS plays an important role where actuators and sensors are linked with the software. The main aim of CPS is to increase the production rate by increasing the efficiency [21] of the system. With the rise of the concept of Industry 4.0, mechanisms have expanded, and an ample amount of importance is given to developing smart systems. Smart and different designs have been considered to handle failures in nonoptimized methods. CPS is very useful in enhancing the flexibility of IoT-based production systems in various domains such as healthcare, agriculture, and financial sectors (banking). The modified human-computer interaction system is developed, which helps us to develop the main ideology of Industry 4.0. Figure 2 is showing Integration of Product Development in Digital and Physical Production.

3.3. Internet of Robotic Things (IoRT). Combining IoRT knowledge from several sources can contribute to a consistent and consistent depiction of the robot world. In a summarized view, IoRT information should only be integrated based on approximate environmental models. In the case of monitoring contacts, for example, in selecting impedance parameters or determining the hazardous control points of the Mobile Robot (MR) to be left for the essential individual, data fusion is significant. The proximity could include proximity IoT like pan/tilt/zoom cameras, stereo cameras, deep camera base, or audio/video feedback systems. Adjustable power and force limitation functions (considerable, high speed, and high payload) can be better adapted to conventional robots (PFL robots). Remote interaction sensors include cameras, stereo cameras, 3D Lidar cameras, and a certified safety sensor. Audio/video recovery: HRC sensors may also be fitted with integrated force/torque or grip proximity sensors.

3.4. Improving Security of Cyber-Physical System Using SDN for Mobile Robotic Industry 4.0. Conceptual Industry 4.0 has a significant impact and many changes in manufacturing, output, and business modeling processes. This ensures mass adaptation, higher productivity, flexibility, speed of production, and improved product quality. Because machines can be quickly set up to suit customer specifications and additives, mass adaptation allows for the production of small lots, even small lots. It encourages innovation because prototypes or new products can be produced quickly without the need for complex retooling or the establishment of new production lines. Therefore, it can produce one product and many variants, with the use of Industry 4.0 technologies reducing its inventories. Besides, the speed at which the product can be manufactured has been improved by reducing the time between designs, digital strategies, and virtual manufacturing modeling. In India, data-driven supply chains can accelerate the production process by approximately 100 percent in terms of delivery time and 60 percent to bring products onto the market. Increased quality is an essential factor in cost reduction and, therefore, competitiveness. If all defects were removed, the cost of scrapping or reprocessing defective products would be saved. The productivity of the various effects of Industry 4.0 can also be increased. Advanced analytics in predictive maintenance programs, which results in increased production and decreased downtime, can help avoid machine failure. Some companies may establish factories where automated robots are manufactured at home without light or heat. Robotics have a significant economic impact with security on industrial applications where increased productivity can drive economic growth, self-driving mobile robots are machines equipped with sensors and feedback to navigate an obstacle-filled environment. It is intended to reduce wear in various industrial components while increasing productivity in the manufacturing sector. Reformist Framework for improving human security with Mobile Robots in Industry 4 [22] has a variety of advantages, including lower floor traffic, flexible flooring, dependability, and self-regulation. The basic requirement to use the autonomous mobile robots is as follows:

(i) Using our approach automatically increasing efficiency and productivity [23]

(ii) Automatic handling of the material

(iii) Automatic increase of the security [24] and safety, reducing the stress and high risk

(iv) Managing the repetitive task with creativity [25]

(v) Reducing the factory traffic in complex conditions

Production control is a crucial vision of Industry 4.0, [26] including improving human security [27] with Mobile Robots in Industry 4.0 [28] factories to fully connect production. Although it does not yet exist because factory systems have not been linked with a unified framework [29], this capability would allow entire production lines to be checked and optimized in different facilities [30]. Many companies see it as a current and future goal, the realization of which will still rely on improved information infrastructure, better data handling security and safety, and significant improvements [31] to improving human security with Mobile Robots in Industry 4.0, as one company has stated, which will be responsible for "automating automation" [32].

Figure 3 shows the analysis of the attacking data for improving human security with Mobile Robots in Industry 4.0 and represents the autonomous sensor with a decision system working.

At employment, efficient human-robot contact is likely to be harmful to both parties. Consider the hazards listed in Figures 4-6 which are potential human-robot interaction risks. Table 3 represents the human-robot interaction.

\section{Proposed Real-Time Attack of Data Classification}

We proposed Gaussian Mixture Model (GMM) [33] with expectation maximization (EM). The internet of things (IoT) avoids obstacles by updating its path in real time, then 


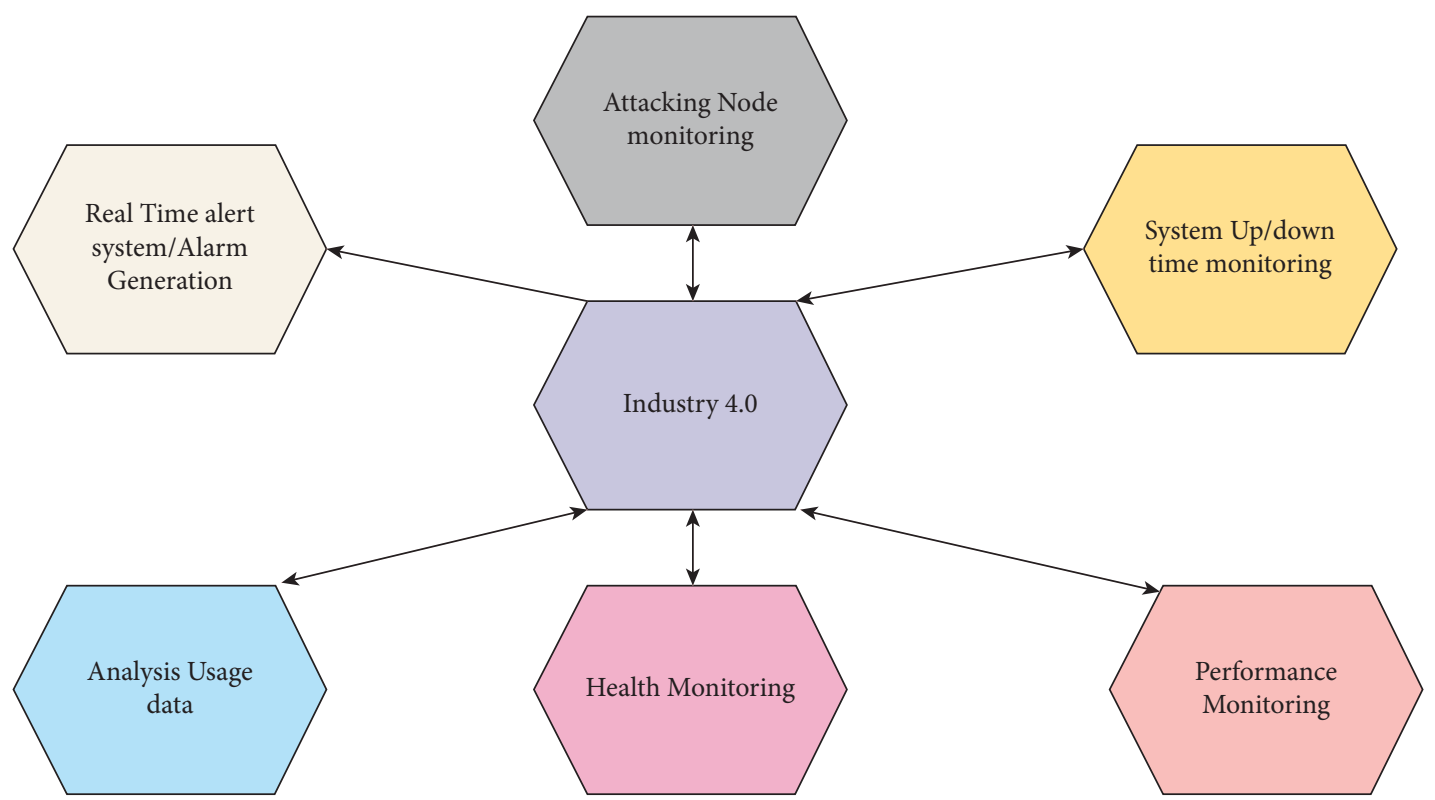

FIGURE 2: Integration of product development in digital and physical production.

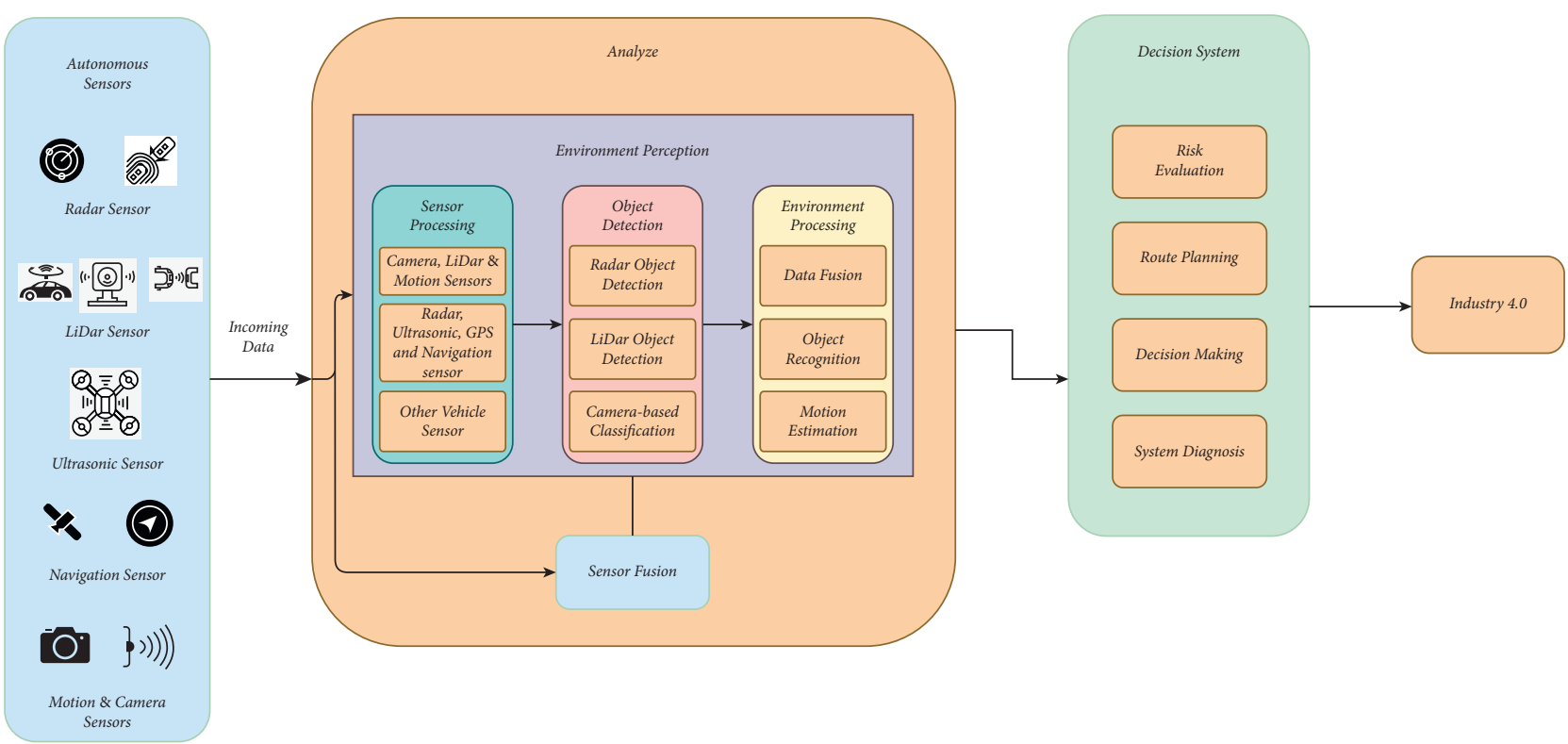

FIGURE 3: Analysis of the attacking data for improving human security with Mobile Robots in Industry 4.0.

bypassing the obstacle and reaching the target point. Anticipating obstacles moving a robot from one area to another is called motion planning. We carried out research on cyber hazards to motion planning. Path and trajectory plans assume that your manipulator's displacement, velocity, and acceleration are all on the same time axis. The trajectory is a path that carries time axis information. Human-robot interaction requires trajectory planning. A static environment with little path planning is their domain-for example, a rapid spreading random tree based on random sampling. Online tracking control is used for most typical robot task pathways. A safety cage protects an operator's workspace. The environment is dynamic, and the problems are predictable and unpredictable. Online real-time collaborative robot trajectory planning based on sensor inputs replaces offline planning. They are predicting path/route utilizing global environmental data like grid maps and random spanning trees. However, their slow solution speed makes them unsuitable for online obstacle avoidance planning. For online obstacle avoidance trajectory planning of robot arms, it uses local environmental data. The operating arm's online trajectory planning system uses potential fields, feedback control, and null space projection. Others strive to avoid cyber-attacks. The planner is described. An $\mathrm{N}$-point dataset comprising joint angles, hand trajectories, or hands object distance vectors can be used to test the 


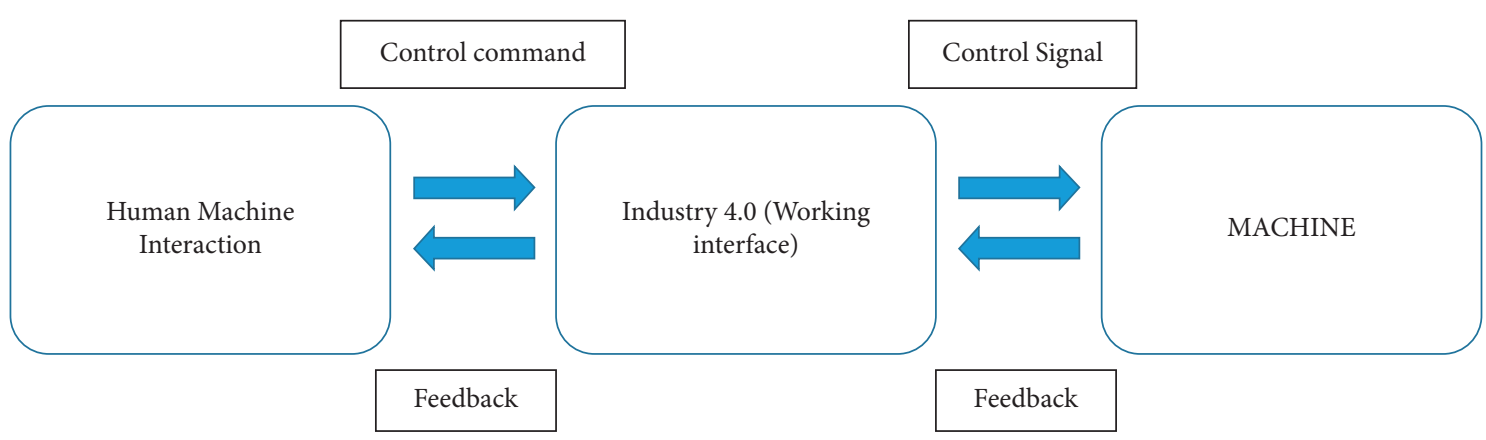

FIgURE 4: Process for human-machine interaction (HMI).

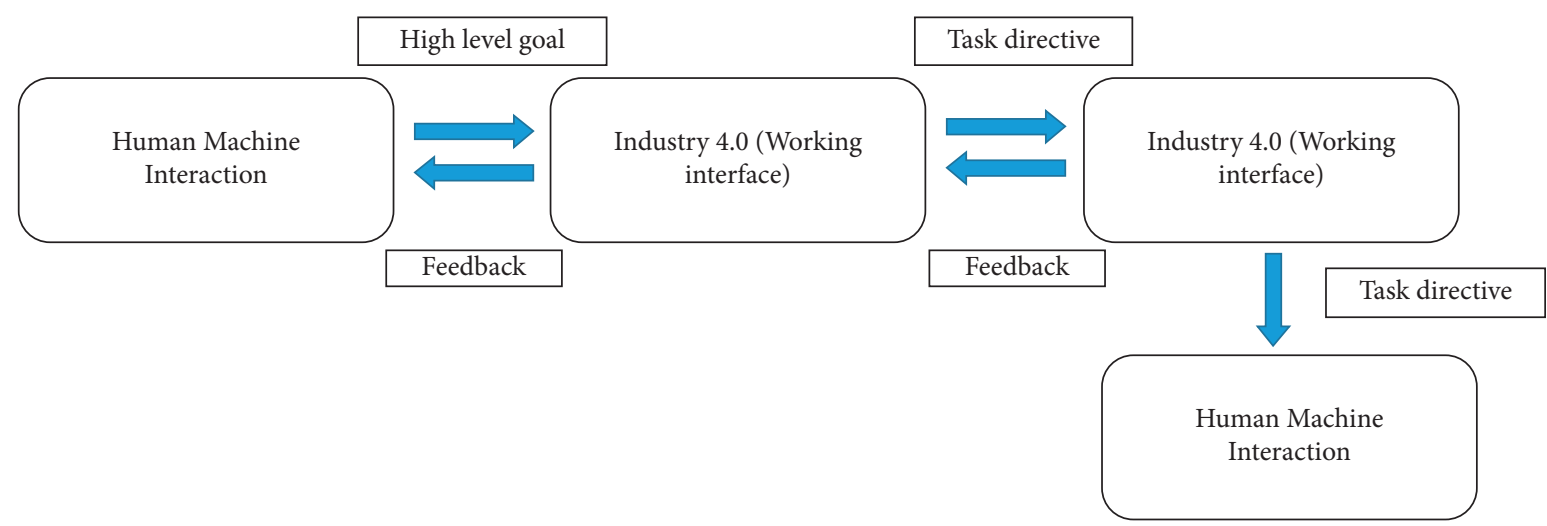

FIgURE 5: Human task interaction.

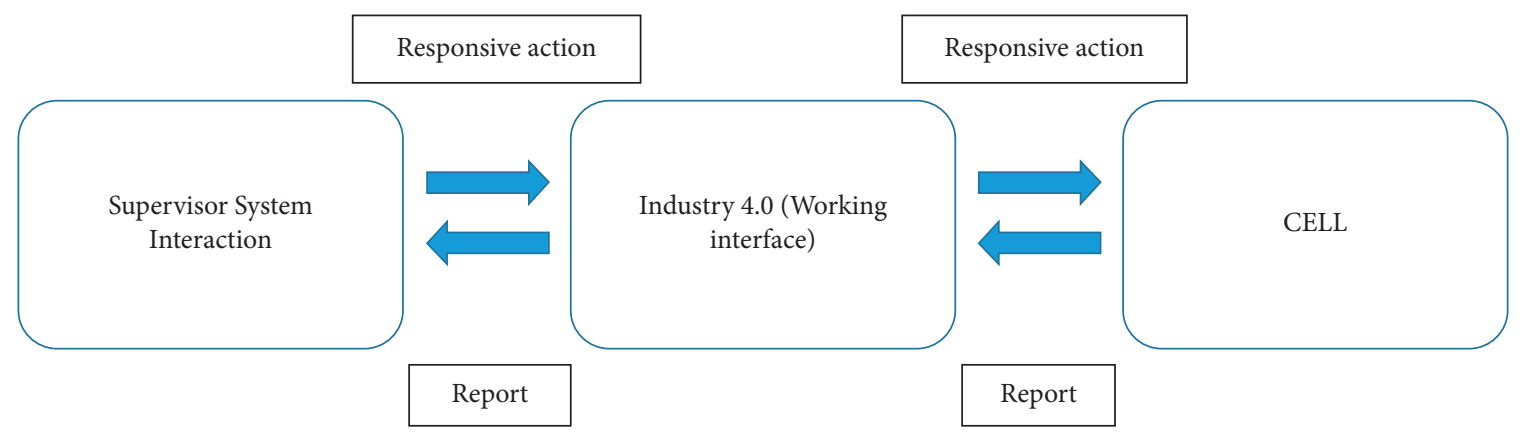

Figure 6: Supervisor system interaction.

TABLE 3: Human-robot interaction.

Human security with mobile robots in Industry 4.0

Explanation

Increasing the gap between human and Mobile Robot operation The Mobile Robot's pathway and its barriers

Safety of hazardous from Mobile Robot Psyche-physiology of the human operator industrial process hazards extra care Inadequate ergonomic solutions for maintenance and operation The work complexity in the working space The influence of operators

Safety of unsafe from the industrial process Errors made by humans while operating robots Creating obstacles for robot sensors to work

Protection from robot control system failure (cyber-attack) 
Gaussian Mixture Model (GMM) with expectation maximization (EM) [34]. In a mixed model with $\mathrm{K}$ components, there is probability density.

\subsection{Automated Manufacturing Internet of Robotic Things} (IoRT) and Cobots. Provide solutions to decreasing talent pools, rising labor costs, and increased competitiveness facing manufacturers today and expanding the workforce with IoRT and cobots that improve worker performance, safety, and retention. Screw driving, low-value shipping, palletizing, and machine maintenance are all jobs that humans are doing. A IoRT increases productivity and quality. People have more time to solve challenging production challenges, improve lean processes, and find creative solutions. Permitting employees to produce greater value while avoiding repetitive and dangerous jobs promotes employee satisfaction. Heavy lifting, overreaching, and repetitive motions are not suited for the human body. Injuries, accidents, and quality difficulties arose from this conflict. A cyber-physical attack may not expose product faults until the end of production. Products that do not undergo postproduction quality testing are in danger of failing. Unidentified or uncorrected structural flaws may enter the market. Structurally deficient, this is seen in design flaws that can go unnoticed by a product's design team. So a new postproduction analysis method is required. Goods made using CT, Raman spectroscopy, and other comparable scanning methods could find problems in products before leaving the plant. SDNs and cyber-physical systems provided an IoRT [35] high-level view of scaling information networks. It is a new and promising answer to the current problematic networking issue (SDN-like isolation of network services and hardware). They argue that network decoupling requires the abstraction of the underlying network technology. This treats the network as a whole. A centralized software-defined network controller is the SDN's brain. Network controller software keeps track of all network device connectivity. An SDN network needs all of these elements. Firewalls, switching, and routing are so segregated. Divvying up a network into these three segments allows for individual improvement. Admins oversee routing, firewalls, and policy_the management plane. Load distribution can increase efficiency and lower costs. The forwarding plane sends packets to network switches (hardware or software). The data plane handles packet header inspection, forwarding to controllers and network ports. Packets directed to devices, not in the flow tables, can be refused or rerouted. Figure 7 is showing the self-driving framework for mobile robots are machines equipped for human security.

It is unquestionably true that the IoRT with SDWNs will significantly benefit industrial businesses looking for an efficient yet realistic networking solution for equipment interconnection.

4.2. Attacking Node Termination for Human Security. Distinguishing cyber-physical components enhanced human-machine interfaces create additional security and interoperability concerns. An increasing number of industrial customers using automatic and semiautomatic assembly processes desire to integrate their workforce. Technically, industrial concerns vary, but they all revolve around safety and security. Once the cyber-layer is breached, SDN-CPS mitigation methods are ineffective in identifying and actively responding IoRT to cyber-attacks. The current development phase aims to boost penetration by understanding the target (CPS) control system. On the right, you can see attack tactics, targets, consequences, and interdependencies among the IoRT with SDN-CPS-security layers [36]. Because SDN-CPS security has numerous system layers, attacks on different system tiers are feasible. Due to the high degree of interdependency between SDN-CPS-security components at different tiers, unexpected repercussions are likely to occur. They can occur at components in various layers or even separate domains (cyber or physical). Ineffective IoRT and CPS assaults have received little attention. The choice of SDN-CPS-security technology poses various technical issues. Calculating the robot's [37] safe distance from the human workers in the production cell is the most difficult. Tight sensor safety distance constraints will be the technological limit. Reliable components and a cyber-security mitigation plan [38] are required to prevent smart cyber-attacks in a networked environment. Even if the system's cyber layer is breached, workers and PCs must be protected. Covering the robot's surfaces to prevent human contact [39] is another challenge. Camera systems require the most safety distance [40]. A speedier human worker means a wider safety gap. Individual sensor data latency rates and network connectivity difficulties can lead to unwanted findings. IoRT provides solutions to eliminate delays caused by using many sensors from various manufacturers [41]. It was determined that worker speed and safety distance are related. This includes risks that can cause system failure and jeopardise human safety.

\section{Results and Discussion}

This section presents all the results and analyses based on a variety of performance measures and evaluating multiple machine learning model [42] (Decision Tree (DT), Random Forest (RF), support vector machine (SVM), and backpropagation neural network (BPNN)) which are identified as the most safe and effective for classifying attack data on IoRT networks [43].

The table illustrates the GMM and EM confusion matrix and two interesting facts. The first is that the data tested is little and the classes are not equally represented. The lack of test data may be affecting the outcomes. Table 4 shows that, except for DT, the other models score poorly compared to RF, SVM, SVMG-RBF, and BPNN. Increase the test data to examine how it affects the RF, SVM, SVMG-RBF, BPNN model's scores [44]. Table 4 shows the effects of increasing IoRT network attacking data. Increasing the test data by $30 \%$ improves recall but not accuracy. After 50\%, the model loses complete recall and precision [45]. Due to a paucity of data, the model's outputs vary greatly. Table 5 represents the comparative analysis traditional model and proposed model [46]. 


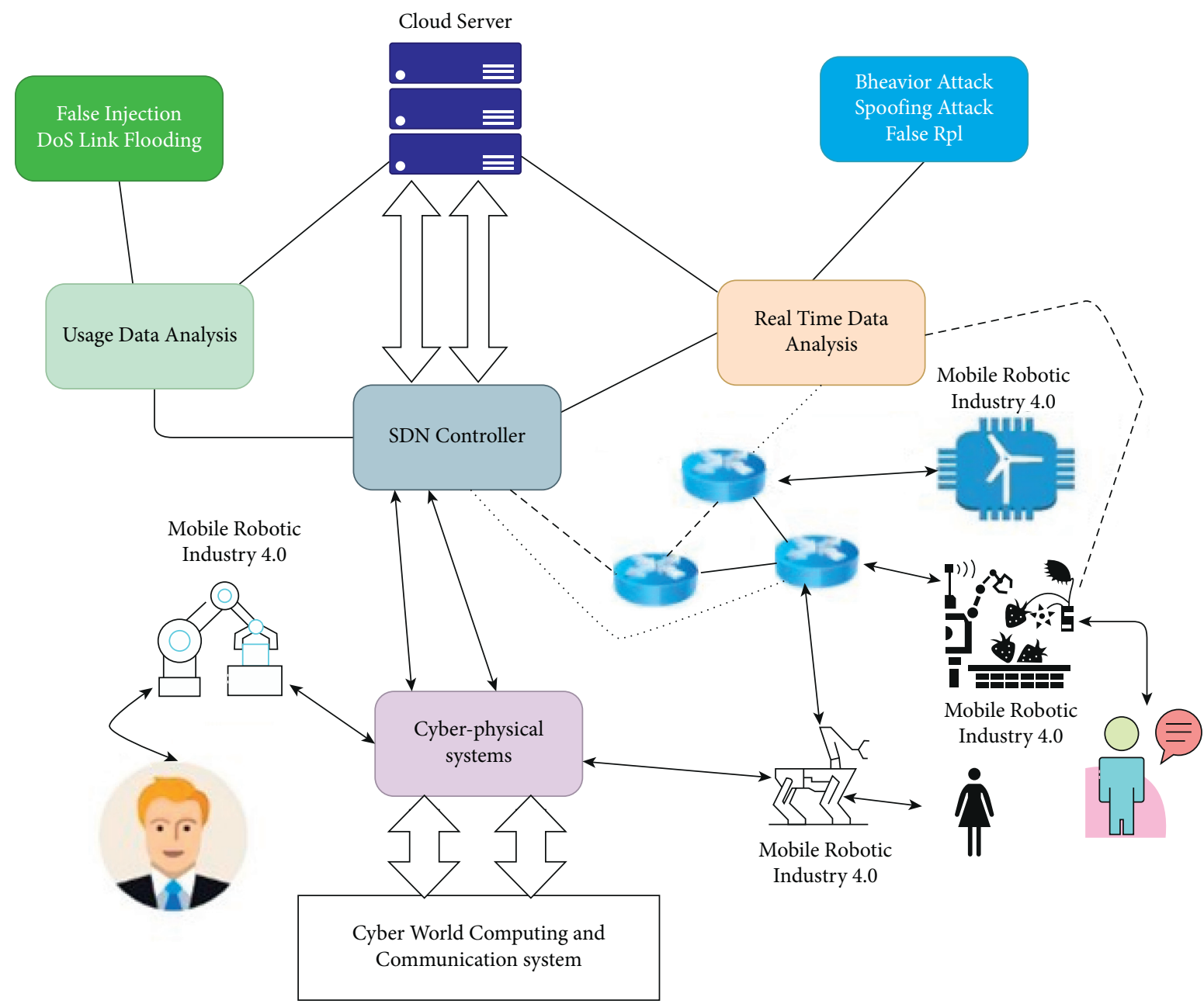

FIGURE 7: Self-driving framework for Mobile Robots are machines equipped for human security.

TABLE 4: Machine learning algorithm for attacking node data classification.

\begin{tabular}{|c|c|c|c|c|}
\hline Algorithm used & Accuracy (\%) & Precision & Recall & F1 score \\
\hline$\overline{\mathrm{DT}}$ & 0.91 & 0.843 & 0.988 & 0.98 \\
\hline $\mathrm{RF}$ & 0.93 & 0.774 & 0.988 & 0.97 \\
\hline SVM & 0.95 & 0.874 & 0.988 & 0.97 \\
\hline SVMG-RBF & 0.95 & 0.866 & 0.988 & 0.99 \\
\hline BPNN & 0.94 & 0.832 & 0.988 & 0.98 \\
\hline Proposed & 0.98 & 0.888 & 0.988 & 0.99 \\
\hline
\end{tabular}

TABLE 5: The comparative analysis traditional model and proposed model.

\begin{tabular}{lrc}
\hline Name algorithm & \multicolumn{1}{c}{ Traditional } & Proposed mr-dfl \\
DT & $\begin{array}{r}\text { Large amounts of memory, a large dataset, } \\
\text { and a lengthy computation time } \\
\text { RF }\end{array}$ & $\begin{array}{c}\text { Reduce the amount of time it takes to compute } \\
\text { and increase accuracy }\end{array}$ \\
SVM & Error evaluation, very slow & Less computation cost \\
SVMG-RBF & Data classification, very slow & Error evaluation, very high \\
BPNN & More computing in large datasets & Data classification, very high response \\
Proposed & Inaccuracy large datasets, significant memory use & Large datasets with excellent precision \\
\end{tabular}




\section{Conclusion}

In this paper, we discussed the general approach to IoRT steering in industrialized backgrounds in which the Mobile Robot's free behaviour and requirements are all automatically based. To achieve a flexible supervision controller using SDN and CPS that safeguards the accurate direction-finding of the robot in the presence of unforeseeable protects. The proposed method provides a general demonstrating structure for specifications through components that are exclusively dependent on the type of jobs achieved by the automation. Our proposed Gaussian Mixture Model (GMM) with expectation maximization (EM) Tussles of improving human security with Mobile Robots in Industry 4.0 architecture proposed in this research part would serve as research directly in cooperation with several robots. In the future work, we plan to include the research's models into an IDS prototype for testing with various data and threats to confirm model multiclass capability.

\section{Data Availability}

The data that support the findings of this study are available on request from the corresponding author.

\section{Conflicts of Interest}

All the authors declare that they do not have any conflicts of interest.

\section{Acknowledgments}

This work was supported by Taif University Researchers Supporting Project Number (TURSP-2020/114), Taif University, Taif, Saudi Arabia.

\section{References}

[1] S. Robla-Gomez, V. M. Becerra, J. R. Llata, E. Gonzalez-Sarabia, C. Torre-Ferrero, and J. Perez-Oria, "Working together: a review on safe human-robot collaboration in industrial environments," IEEE Access, vol. 5, pp. 26754-26773, 2017.

[2] M. Aazam, S. Zeadally, and K. A. Harras, "Deploying fog computing in industrial internet of things and industry 4.0," IEEE Transactions on Industrial Informatics, vol. 14, no. 10, pp. 4674-4682, 2018.

[3] M. C. Lucas-Estañ, B. Coll-Perales, and J. Gozalvez, "Redundancy and diversity in wireless networks to support mobile industrial applications in industry 4.0," IEEE Transactions on Industrial Informatics, vol. 17, no. 1, pp. 311-320, 2021.

[4] T. P. Raptis, A. Passarella, and M. Conti, "Data management in industry 4.0: state of the art and open challenges," IEEE Access, vol. 7, pp. 97052-97093, 2019.

[5] A. G. C. Gonzalez, M. V. S. Alves, G. S. Viana, L. K. Carvalho, and J. C. Basilio, "Supervisory control-based navigation architecture: a new framework for autonomous robots in industry 4.0 environments," IEEE Transactions on Industrial Informatics, vol. 14, no. 4, pp. 1732-1743, 2018.

[6] J. Kumar, P. Bedi, S. B. Goyal, A. Shrivastava, and S. Kumar, "Novel algorithm for image classification using cross deep learning technique," IOP Conference Series: Materials Science and Engineering, vol. 1099, Article ID 012033, 2021.

[7] B. Chen, J. Wan, L. Shu, P. Li, M. Mukherjee, and B. Yin, "Smart factory of industry 4.0: key technologies, application case, and challenges," IEEE Access, vol. 6, pp. 6505-6519, 2018.

[8] J. Wan, S. Tang, Q. Hua, D. Li, C. Liu, and J. Lloret, "Contextaware cloud robotics for material handling in cognitive industrial internet of things," IEEE Internet of Things Journal, vol. 5, no. 4, pp. 2272-2281, 2018.

[9] E. Åblad, D. Spensieri, R. Bohlin, and J. S. Carlson, "Intersection-free geometrical partitioning of multirobot stations for cycle time optimization," IEEE Transactions on Automation Science and Engineering, vol. 15, no. 2, pp. 842-851, 2018.

[10] M. Agarwal, S. Biswas, C. Sarkar, S. Paul, and H. S. Paul, "Jampacker: an efficient and reliable robotic bin packing system for cuboid objects," IEEE Robotics and Automation Letters, vol. 6, no. 2, pp. 319-326, 2021.

[11] T.-H. Chiang, Z.-H. Sun, H.-R. Shiu, K. C.-J. Lin, and Y.-C. Tseng, "Magnetic field-based localization in factories using neural network with robotic sampling," IEEE Sensors Journal, vol. 20, no. 21, pp. 13110-13118, 2020.

[12] E. Oztemel and S. Gursev, "Literature review of industry 4.0 and related technologies," Journal of Intelligent Manufacturing, vol. 31, no. 1, pp. 127-182, 2020.

[13] A. S. Rajawat, S. Jain, and K. Barhanpurkar, "Fusion protocol for improving coverage and connectivity WSNs," IET Wireless Sensor Systems, vol. 11, no. 4, 2021.

[14] L. D. Evjemo, T. Gjerstad, E. I. Grøtli, and G. Sziebig, "Trends in smart manufacturing: role of humans and industrial robots in smart factories," Current Robotics Reports, vol. 1, no. 2, pp. 35-41, 2020.

[15] M. A. Bahrin, M. F. Othman, N. H. Nor Azli, and M. F. Talib, "Industry 4.0: a review on industrial automation and robotic," Jurnal Teknologi, vol. 78, 2016.

[16] A. G. Gonzalez, M. V. Alves, G. S. Viana, L. K. Carvalho, and J. C. Basilio, "Supervisory control-based navigation architecture: a new framework for autonomous robots in industry 4.0 environments," IEEE Transactions on Industrial Informatics, vol. 14, no. 4, pp. 1732-1743, 2017.

[17] E. Sanz, J. Blesa, and V. Puig, "BiDrac industry 4.0 framework: application to an automotive paint shop process," Control Engineering Practice, vol. 109, Article ID 104757, 2021.

[18] M. Di Castro, M. Ferre, and A. Masi, "CERNTAURO: a modular architecture for robotic inspection and telemanipulation in harsh and semi-structured environments," IEEE Access, vol. 6, pp. 37506-37522, 2018.

[19] A. Kattepur and B. Purushotaman, "RoboPlanner: a pragmatic task planning framework for autonomous robots," Cognitive Computation and Systems, vol. 2, no. 1, pp. 12-22, 2020.

[20] A. K. Jain and M. Svetlik, "An open-source framework for last mile delivery with heterogeneous robots," in Proceedings of the AAAI 2021 Spring Symposium Series, Machine Learning for Mobile Robot Navigation in the Wild, Palo Alto, CA, USA, 2021.

[21] P. Kumar Shukla, P. Sharma, P. Rawat, J. Samar, R. Moriwal, and M. Kaur, "Efficient prediction of drug-drug interaction using deep learning models," IET Systems Biology, vol. 14, no. 4, pp. 211-216, 2020.

[22] Q. Chen, B. Heydari, and M. Moghaddam, "Levering task modularity in reinforcement learning for adaptable industry 4.0 automation," Journal of Mechanical Design, vol. 143, no. 7 , pp. 1-35, 2021. 
[23] H. Fatorachian and H. Kazemi, "Impact of industry 4.0 on supply chain performance," Production Planning \& Control, vol. 32, no. 1, pp. 63-81, 2021.

[24] A. S. Rajawat, U. Dwivedi, D. C. Jain, and A. R. Upadhyay, "Efficient web data classification techniques using semi-supervise learning algorithm," in Proceedings of the 5th ACM COMPUTE Conference on Intelligent \& Scalable System Technologies, pp. 1-8, Pune, India, January 2012.

[25] M. Ammar, A. Haleem, M. Javaid, R. Walia, and S. Bahl, "Improving material quality management and manufacturing organizations system through industry 4.0 technologies," Materials Today: Proceedings, vol. 45, no. 4, 2021.

[26] B. Ralph and M. Stockinger, "Digitalization and digital transformation in metal forming: key technologies, challenges and current developments of industry 4.0 applications," in Proceedings of the XXXIX. Colloquium on Metal Forming, Leoben, Austria, March 2021.

[27] A. S. Rajawat and A. R. Upadhyay, "Web personalization model using modified S3VM algorithm for developing recommendation process," in Proceedings of the 2nd International Conference on Data, Engineering and Applications (IDEA), pp. 1-6, Bhopal, India, February 2020.

[28] P. Prabhu, S. Raj, and A. Roy Chowdhury, "Interlinking industry 4.0 and academia through robotics and automation: an Indian perspective," Convergence of Digitalization on $5 G$ Edge AI Computing, IntechOpen Limited, London, UK, 2020.

[29] S. V. Lobova, N. V. Bykovskaya, I. M. Vlasova, and O. V. Sidorenko, "Successful experience of formation of industry 4.0 in various countries," in Industry 4.0: Industrial Revolution of the 21st Century, pp. 121-129, Springer, Berlin, Germany, 2019.

[30] R. Bhatt, P. Maheshwary, P. Shukla, P. Shukla, M. Shrivastava, and S. Changlani, "Implementation of fruit fly optimization algorithm (FFOA) to escalate the attacking efficiency of node capture attack in wireless sensor networks (WSN)," Computer Communications, vol. 149, pp. 134-145, 2020.

[31] R. Rajkumar, I. Lee, L. Sha, and J. Stankovic, "Cyber-physical systems: the next computing revolution," in Proceedings of the Design Automation Conference, pp. 731-736, IEEE, Anaheim, CA, USA, June 2010.

[32] R. Gervasi, L. Mastrogiacomo, and F. Franceschini, "A conceptual framework to evaluate human-robot collaboration," International Journal of Advanced Manufacturing Technology, vol. 108, no. 3, pp. 841-865, 2020.

[33] L. Romeo, A. Petitti, R. Marani, and A. Milella, "Internet of robotic things in smart domains: applications and challenges," Sensors, vol. 20, no. 12, p. 3355, 2020.

[34] A. Ghosh, D. Alonso Paredes Soto, S. M. Veres, and A. Rossiter, "Human robot interaction for future remote manipulations in industry 4.0," IFAC, vol. 53, no. 2, pp. 10223-10228, 2020.

[35] Z. Gao, W. Tom, I. Singh, A. Gadhrri, and R. Schmidt, "From industry 4.0 to robotics $4.0-\mathrm{a}$ conceptual framework for collaborative and intelligent robotic systems," Procedia Manufacturing, vol. 46, pp. 591-599, 2020.

[36] A. Khalid, P. Kirisci, Z. Ghrairi, K.-D. Thoben, and J. Pannek, "Towards implementing safety and security concepts for human-robot collaboration in the context of industry 4.0," in Proceedings of the 39th International MATADOR Conference on Advanced Manufacturing, Manchester, UK, July 2017.

[37] S. Bragança, E. Costa, I. Castellucci, and P. M. Arezes, "A brief overview of the use of collaborative robots in industry 4.0: human role and safety," Occupational and Environmental
Safety and Health, Springer, Cham, Germany, pp. 641-650, 2019.

[38] Y. Li, S. Carabelli, E. Fadda, D. Manerba, R. Tadei, and O. Terzo, "Machine learning and optimization for production rescheduling in industry 4.0," International Journal of Advanced Manufacturing Technology, vol. 110, no. 9-10, pp. 2445-2463, 2020.

[39] Z. Zeng, W. Sun, W. Wu, M. Xue, and L. Qian, "An efficient path planning algorithm for mobile robots," in Proceedings of the IEEE 2019 IEEE 15th International Conference on Control and Automation (ICCA), pp. 487-493, Edinburgh, UK, July 2019.

[40] H. Li and A. V. Savkin, "An algorithm for safe navigation of mobile robots by a sensor network in dynamic cluttered industrial environments," Robotics and Computer-Integrated Manufacturing, vol. 54, pp. 65-82, 2018.

[41] D. Singh, "Human emotion recognition system," International Journal of Image, Graphics and Signal Processing, vol. 4, no. 8, pp. 50-56, 2012.

[42] H. S. Pannu, D. Singh, and A. K. Malhi, "Multi-objective particle swarm optimization-based adaptive neuro-fuzzy inference system for benzene monitoring," Neural Computing and Applications, vol. 31, no. 7, pp. 2195-2205, 2019.

[43] H. S. Pannu, D. Singh, and A. K. Malhi, "Improved particle swarm optimization based adaptive neuro-fuzzy inference system for benzene detection," Clean Soil Water, vol. 46, no. 5, Article ID 1700162, 2018.

[44] P. K. Shukla, M. Agrawal, and A. U. Khan, "Stock price prediction using technical indicators: a predictive model using optimal deep learning," International Journal of Recent Technology and Engineering (IJRTE), vol. 8, no. 2, pp. 22972305, 2019.

[45] S. B. Goyal, P. Bedi, J. Kumar, and V. Varadarajan, "Deep learning application for sensing available spectrum for cognitive radio: an ECRNN approach," Peer-to-Peer Networking and Applications, vol. 14, no. 5, 2021.

[46] P. Bedi, S. B. Goyal, R. Sharma, D. K. Yadav, and M. Sharma, "Smart model for big data classification using deep learning in wireless body area networks," in Micro-Electronics and Telecommunication Engineering. Lecture Notes in Networks and Systems, D. K. Sharma, L. H. Son, R. Sharma, and K. Cengiz, Eds., vol. 179, Springer, Singapore, 2021. 